\title{
Relationship of Oct-4 to malignant stage: a meta-analysis based on 502 positive/high Oct-4 cases and 522 negative/low case- free controls
}

\author{
Beilong Zhong ${ }^{1, *}$, Yan Lin $2, *$, Yingrong Lai ${ }^{3, *}$, Fangfang Zheng ${ }^{4}$, Xiaobin Zheng ${ }^{5}$, \\ Rijiao Huang ${ }^{6}$, Weilin Yang ${ }^{7}$, Zhenguang Chen ${ }^{7,8,9, *}$ \\ ${ }^{1}$ Department of Thoracic Surgery, The Fifth Affiliated Hospital, Sun Yat-sen University, Zhuhai, Guangdong 519000, China \\ ${ }^{2}$ Department of Breast Disease, Peking Union Medical College Hospital, Peking Union Medical College, Beijing 100730, China \\ ${ }^{3}$ Department of Pathology, The First Affiliated Hospital, Sun Yat-sen University, Guangzhou, Guangdong 510080, China \\ ${ }^{4}$ Department of Pediatrics, The Fifth Affiliated Hospital, Sun Yat-sen University, Zhuhai, Guangdong 519000, China \\ ${ }^{5}$ Department of Respiratory Medicine, The Fifth Affiliated Hospital, Sun Yat-sen University, Zhuhai, Guangdong 519000, \\ China \\ ${ }^{6}$ Department of Clinical Laboratory, The Fifth Affiliated Hospital, Sun Yat-sen University, Zhuhai, Guangdong 519000, China \\ ${ }^{7}$ Department of Cardiothoracic Surgery of East Division, the First Affiliated Hospital, Sun Yat-sen University, Guangzhou, \\ Guangdong 510080, China \\ ${ }^{8}$ Department of Thoracic Surgery, The First Affiliated Hospital, Sun Yat-sen University, Guangzhou, Guangdong 510080, \\ China \\ ${ }^{9}$ Lung Cancer Research Center of Sun Yat-sen University, Guangzhou, Guangdong 510080, China \\ *These authors have contributed equally to this work \\ Correspondence to: \\ Zhenguang Chen, e-mail: chenzhenguang@yahoo.com \\ Keywords: Oct-4, cancer staging, TNM staging, cancer grade of differentiation, meta-analysis \\ Received: May 28, $2015 \quad$ Accepted: October 28, $2015 \quad$ Published: November 09, 2015
}

\section{ABSTRACT}

Background: Octamer 4 (Oct-4), an important member of the POU domain transcription factor family, has been suggested to function as a master switch during differentiation of human somatic cells and more recently has come to be linked with neoplastic properties. The aim of this study was to evaluate the relationship between Oct-4 and cancer stage using a meta-analysis approach.

Materials and Methods: Relevant articles published as of May 2015 were retrieved from the following databases: PubMed, ISI Web of Knowledge, Embase, and Chinese National Knowledge Infrastructure (CNKI). The strengths of relationship for outcomes of interest were estimated based on odds ratios (ORs) and $95 \%$ confidence intervals (CIs).

Results: A total of 11 articles on Oct-4 and cancer staging that collectively included 502 positive/high Oct-4 cases and 522 negative/low case-free controls were chosen. Positive/high Oct-4 was significantly associated with cancer stage in several kinds of cancer. Specifically, positive/high Oct-4 was associated with cancer stage III/IV (fixed effects: $O R=1.53,95 \% \mathrm{CI}=1.12-2.10$ ), primary tumor $\left(T_{3-4}\right)$ (random effects: $\mathrm{OR}=1.93,95 \% \mathrm{CI}=0.99-3.77$ ), and cancer grade of differentiation (intermediate-poor) (random effects: $\mathrm{OR}=3.45,95 \% \mathrm{CI}=1.5-7.61$ ).

Conclusion: These findings suggest that positive/high Oct-4 is more strongly linked to stage III/IV cancer and cancer grade of differentiation, and is correlated with malignant characteristics that lead to poor prognosis in different types of cancer, especially in Asian. Given variability related to ethnicity and differences in cancer types, additional studies are warranted to establish the generalizability of our findings. 


\section{INTRODUCTION}

Expression signature of the stemness state of primary tumors may represent a specific approach for identifying patients who are most likely to suffer recurrence or develop metastases. [1] Actually the overexpression of several genes regulating stem cell properties has been documented in cancer tissues suggesting a possible prognostic role. For example, positive/high nestin may be more strongly linked to advanced cancer stage and correlated with malignant characteristics that lead to poor prognosis in different cancers, especially lung cancer. [2] Aldehyde dehydrogenase 1 activity marked breast cancer cells enriched for stem cell properties, at the same time, a prognostic role of SOX2 seemed to be a more suitable marker of early recurrence in breast cancer. $[1,3]$ Other data suggested that increased level of ZFP57 and decreased expression of CPT1A and CPT1C were associated with high grade glioblastoma. [4] On the other hand, Octamer 4 (Oct-4), a member of the POUdomain transcription factor family, is normally expressed in both adult and embryonic stem cells, $[5,6]$ but it has also been found to be expressed in both mouse and primordial germ cells. [7, 8] Extensive investigations have revealed that Oct-4 is expressed in some cancer cell types, such as breast, prostate, hypopharyngeal, bladder, lung, esophageal, and hepatocellular cancer. [9-15]

Recent reports have demonstrated that Oct- 4 is not only involved in controlling the maintenance of stem cell pluripotency, it is also responsible for the unlimited proliferative potential of stem cells, suggesting that Oct4 serves as a master switch during differentiation of human somatic cells. [16-18] Moreover, overexpression of Oct- 4 increases the malignant potential of tumors, and downregulation of Oct-4 in tumor cells inhibits tumor growth, suggesting that Oct-4 might contribute on maintaining the survival of cancer cells. $[19,20]$ Some characteristics of Oct-4 seemed involve as a functional switch related to the "stemness" of the cancer or cancer stem cells, which had specific ability to give rise to all cell types found in a particular cancer sample, and persist in tumors as a distinct population and cause metastasis by giving rise to new tumors.

Some subsequent studies of Oct-4 have also reported a link between Oct- 4 and malignant characteristics, and suggested that abundant Oct-4 expression correlates with greater malignancy and poor prognosis in different cancer types. To date, however, there has not been an affirmative conclusion about the association of Oct-4 with malignancy. Thus, clarifying the contribution of Oct4 to malignant properties requires an evaluation of the relationship between Oct-4 and cancer stage.

Although several studies have addressed this relationship, including Zhang et al., [21] Ge et al., [22] Dong et al., [23] Chen et al., [24] He et al., [25] Zhang et al., [26] Li et al., [27] Takako et al. [28] and Ji et al., [29] only some reported a strong association between positive/ high Oct-4 and cancer stage. Because the limited sample size of individual studies limits their statistical power, it is important to summarize otherwise inconclusive results across multiple studies to provide evidence for an association of positive/high Oct-4 with cancer stage.

Cancer stage is determined using the TNM (primary tumor, lymph nodes, and distant metastasis) classification system, which has been in worldwide use across all medical specialties for five decades. [30] The TNM system is based on the anatomic extent of the tumor as determined clinically and, in most instances, histopathologically. $[30,31]$ TNM Staging Standard is a regularly updated manual for the classification of malignant tumors, published by the American Joint Committee on Cancer (AJCC) and the Union for International Cancer Control (UICC) for Malignant Tumors (4th edition). Currently, TNM staging is widely used throughout the world because of its simplicity and prognostic ability. In oncology, TNM staging can facilitate diagnosis, prognosis, treatment, and other clinical decisions. With the 7th edition of AJCC on the National Comprehensive Cancer Network (NCCN) guidelines of esophageal cancer, cancer grade of differentiation was added to the TNM staging system for the evaluation of esophageal cancer stage.

Meta-analysis is a statistical technique for combining results from different studies to produce a single estimate of the major effect with enhanced precision. [32] In order to elucidate the association of positive/high Oct-4 with cancer stage, we carried out a meta-analysis of all eligible studies. Furthermore, we guided subgroup analyses by stratification according to the primary tumor and cancer grade of differentiation.

\section{RESULTS}

\section{Study characteristics}

Our initial search strategy identified 138 potentially relevant studies. After reviewing the title and abstract, a total of 43 articles consistent with our search criteria were preliminarily chosen for further detailed evaluation. After careful screening, 21 studies were excluded because the data were insufficient for our analysis. Of these 21 studies, 11 did not focus on Oct-4 and cancer staging. Ultimately, 11 studies on Oct- 4 and cancer staging were deemed eligible for the final analysis; collectively, these studies included a total of 502 positive/high Oct-4 cases and 522 negative/low case-free controls. The characteristics of the included studies are listed in Table 1. All studies were related to clinical research, including three lung cancer studies, two esophageal squamous cell carcinoma studies, one hypopharyngeal squamous cell carcinoma study, one hepatocellular cancer study, one head and neck squamous cell carcinoma study, one ovarian cancer study, one breast 
Table 1: Characteristics of the studies included in the meta-analysis

\begin{tabular}{|c|c|c|c|c|c|c|}
\hline First author & Year & Country & Ethnicity & Cancer type & $\begin{array}{c}\text { Total number } \\
\text { of patients }\end{array}$ & $\begin{array}{c}\text { Median age } \\
\text { (years) }\end{array}$ \\
\hline Zhang & 2010 & China & Asian & LA & 112 & 57 \\
\hline $\mathrm{Ge}$ & 2010 & China & Asian & HSCC & 85 & 60 \\
\hline Chen & 2012 & China & Asian & Lung cancer & 113 & 57.2 \\
\hline Dong & 2012 & China & Asian & $\begin{array}{c}\text { Hepatocellular } \\
\text { cancer }\end{array}$ & 152 & NR \\
\hline $\mathrm{He}$ & 2012 & China & Asian & ESCC & 153 & 56.4 \\
\hline Lilly & 2012 & America & Caucasian & HNSCC & 110 & NR \\
\hline $\mathrm{Li}$ & 2012 & China & Asian & ESCC & 50 & 62 \\
\hline Zhang & 2013 & China & Asian & Ovarian cancer & 74 & NR \\
\hline $\mathrm{Li}$ & 2013 & China & Asian & Lung cancer & 102 & NR \\
\hline $\mathrm{Ji}$ & 2014 & China & Asian & $\mathrm{CSCC}$ & 43 & 49.6 \\
\hline Takako & 2014 & Japan & Asian & Breast cancer & 93 & NR \\
\hline
\end{tabular}

Abbreviations: CSCC, cervical squamous cell cancer; ESCC, esophageal squamous cell carcinoma; HSCC, hypopharyngeal squamous cell carcinoma; HNSCC, head and neck squamous cell carcinoma; LA, lung adenocarcinoma; NR, not reported.

cancer study, and one cervical squamous cell cancer study. All cancers were confirmed pathologically. The study selection process is shown in Figure 1.

\section{Publication bias}

Because part of the information was not complete in two studies, a funnel plot analysis was performed from nine studies to assess publication bias (Figure 2A). It showed no obvious asymmetry in any cancer staging parameter (positive/high Oct-4 versus negative/low Oct-4), and the results revealed no publication bias $(P>0.05)$.

\section{Meta-analysis results}

TNM staging data relating to primary tumor, lymph node involvement and cancer grade of differentiation were extracted. The frequency of primary tumor, lymph node involvement, cancer grade of differentiation and cancer stage in positive/high and negative/low Oct-4 groups are shown in detail in Tables 2 and 3.

Nine articles that collectively included 418 cases and 438 controls were used to evaluate the relationship between Oct-4 and cancer stage. When all eligible studies were pooled in the meta-analysis, there was evidence of an association between positive/high Oct-4 and cancer stage III/IV in different cancers. As show in Figure 2B, significant main effects were observed between Oct-4 and cancer stage III/IV (positive/high Oct-4 versus negative/ low Oct-4: $\mathrm{OR}=1.53,95 \% \mathrm{CI}=1.12-2.10, P=0.008$ ).

Seven articles that collectively included 316 cases and 381 controls were used to evaluate the relationship between Oct-4 and primary tumor. As shown in Figure 3A, an analysis stratified by primary tumor $\left(\mathrm{T}_{3-4}\right)$ showed that a significant main effect remained (positive/high Oct-4 versus negative/low Oct-4: $\mathrm{OR}=1.93,95 \% \mathrm{CI}=0.99$ $-3.77, P=0.05)$. However, as shown in Figure $3 \mathrm{~B}$, an analysis stratified by lymph nodes $\left(\mathrm{N}_{1-2}\right)$ showed that there was no significant main effect remained (positive/ high Oct- 4 versus negative/low Oct- 4 : OR $=0.99,95 \%$ $\mathrm{CI}=0.53-1.83, P=0.97$ ).

Seven articles that collectively included 312 cases and 372 controls were used to evaluate the relationship between Oct-4 and cancer grade of differentiation (intermediate-poor). As show in Figure 3C, an analysis stratified by cancer grade of differentiation showed that the main effect remained (positive/high Oct-4 versus negative/ low Oct-4: $\mathrm{OR}=3.45,95 \% \mathrm{CI}=1.56-7.61, P=0.002)$.

\section{Tests of heterogeneity}

Statistically significant heterogeneity was observed between trials of the following analyses using the $\mathrm{Q}$ statistic: Oct-4 with cancer stage III/IV, positive/high Oct4 versus negative/low Oct-4 $\left(P=0.19, I^{2}=29 \%\right)$; Oct4 with primary tumor $\left(\mathrm{T}_{3-4}\right)$, positive/high Oct-4 versus negative/low Oct-4 $\left(P=0.01, I^{2}=64 \%\right)$; Oct-4 with cancer grade of differentiation, positive/high Oct-4 versus negative/low Oct-4 $\left(P=0.003, I^{2}=69 \%\right)$. There was no obvious heterogeneity in Oct-4 versus cancer stage; thus, the analysis was performed using a fixed-effects model. For primary tumor and cancer grade of differentiation, which exhibited moderate heterogeneity, analyses were performed using a random-effects model. 


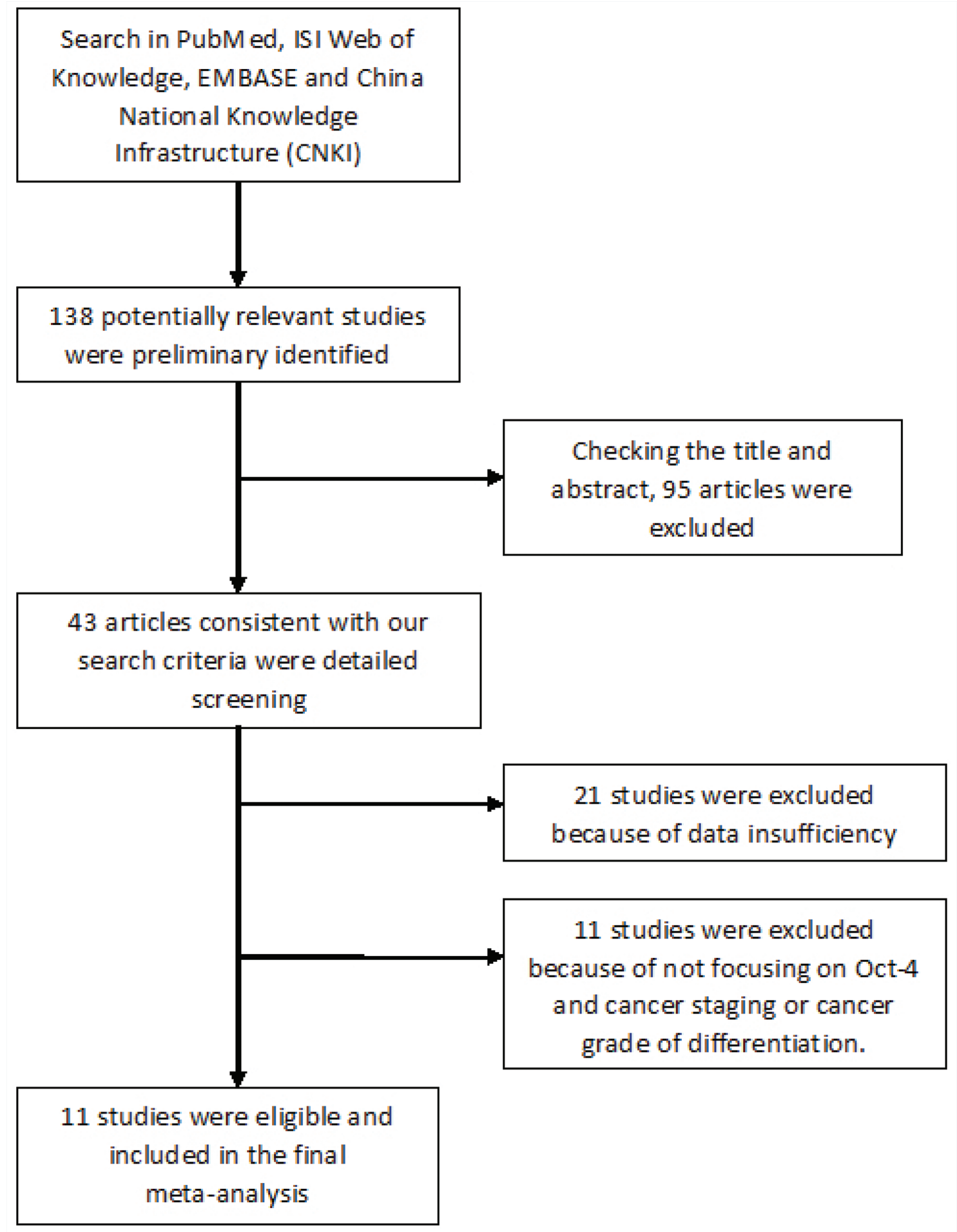

Figure 1: Flow chart of study selection.

\section{DISCUSSION}

TNM staging is determined based on the outcomes of physical examination, biopsy, and imaging tests. [31, 34]. In clinical application, a pathology report forms the basis of TNM staging. In addition to anatomic tumor categories, most sites and tumor types have a TNM staging system, $[31,32]$ with the anatomic extent of the tumor determining the cancer stage. Predicting survival probability and distinguishing among stages requires that each stage be homogeneous. Stages I to III correspond to progressively higher mortality due to localized and regional cancer. 

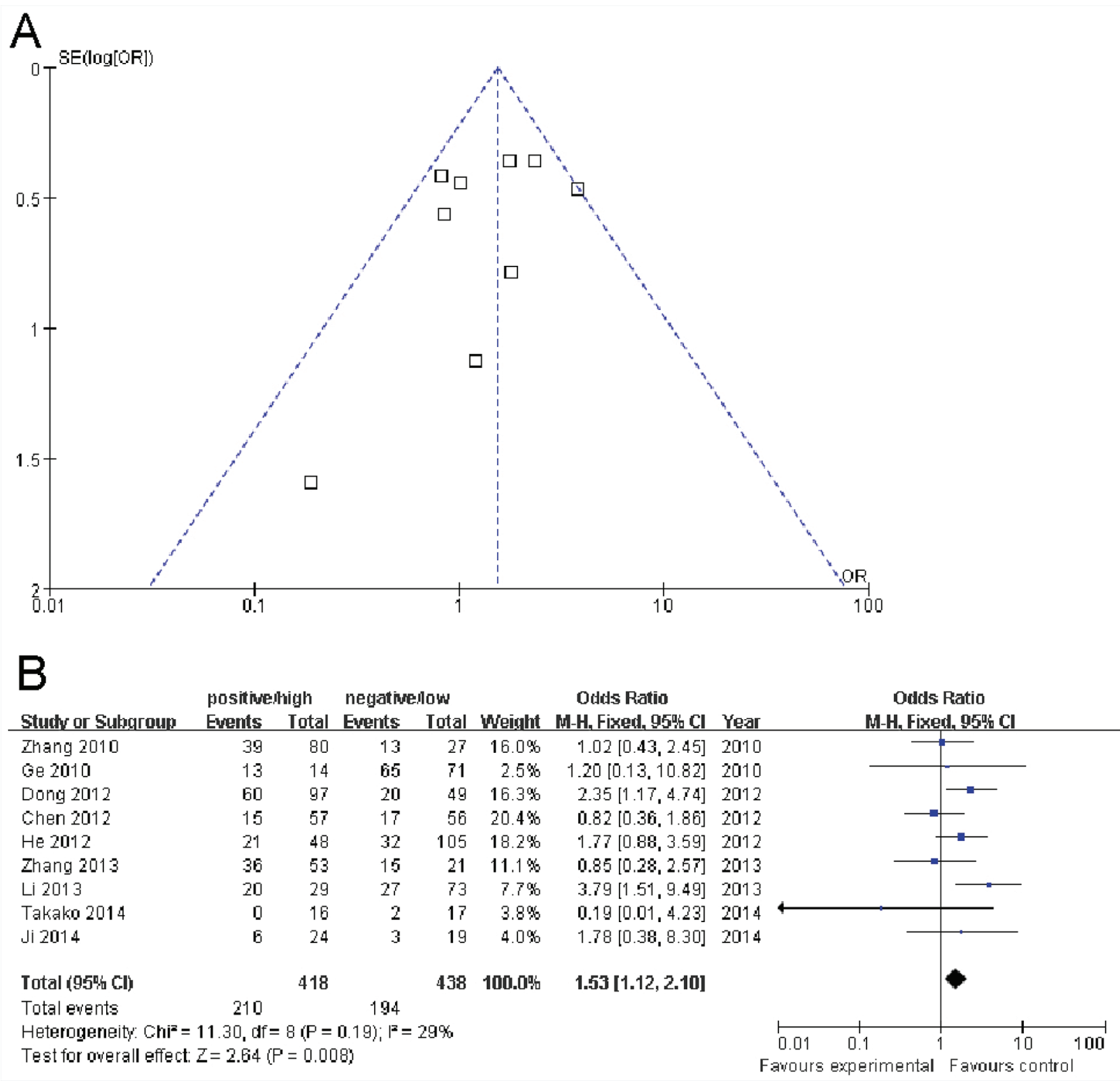

Figure 2: A. Funnel plot assessment of evidence for publication bias from nine studies (positive/high Oct-4 vs. negative/low Oct-4 in cancer staging). B. Forest plots of Oct-4 and cancer stage III/IV in all cases (positive/high Oct-4 vs. negative/low Oct-4). The squares and horizontal lines correspond to the study-specific OR and 95\% CI. The area of the squares reflects the weight (inverse of the variance). The diamond represents the summary OR and $95 \%$ CI.

Stage IV is equivalent to systemic metastases. An accurate staging system is crucial for obtaining prognoses and guiding physicians in treatment strategies. [35]

Oct-4 is an important transcription factor that contributes on pluripotent stem cells. [36] Reports have shown that sustained expression of Oct-4 in epithelial tissues leads to dysplastic changes through inhibition of cellular differentiation; these actions, which are similar to the effects of Oct-4 in some progenitor cells, suggest that Oct-4 may serve the genesis of tumors. [37] However, the mechanisms by which Oct-4 acts during tumor progression, and notably the relationship between Oct-4 and cancer stage, have remained poorly understood.

Several clinical studies, including those by Ge et al., [22] Dong et al., [23] He et al., [25] Li et al., [27] and Ji et al. [29], have reported that positive/high Oct-4 s strongly and significantly associated with cancer stage. And it has been found that the expression of Oct-4 in malignant pleural effusion significantly related to distant metastasis and stage, as well as inversely correlated with patient survival. [38] The aim of the current study was to elucidate the relationship between Oct-4 and cancer stage. Our meta-analysis provided 
Table 2: Positive/high Oct-4 and negative/low Oct-4 frequency in cases and controls: TNM stage

FA
\begin{tabular}{|l|c|c|c|c|c|c|c|c|c|c|c|c|c|c|c|}
\hline & Years & \multicolumn{1}{|c|}{ Cases (positive/high) } & \multicolumn{1}{c|}{ Controls (negative/low) } \\
\hline Zhang & 2010 & 80 & $\mathrm{NR}$ & $\mathrm{NR}$ & $\mathrm{NR}$ & $\mathrm{NR}$ & 41 & 39 & 27 & $\mathrm{NR}$ & $\mathrm{NR}$ & $\mathrm{NR}$ & $\mathrm{NR}$ & 14 & 13 \\
\hline Ge & 2010 & 14 & 3 & 11 & 7 & 7 & 1 & 13 & 71 & 18 & 53 & 59 & 12 & 6 & 65 \\
\hline Chen & 2012 & 57 & 35 & 22 & 24 & 33 & 42 & 15 & 56 & 43 & 13 & 22 & 34 & 39 & 17 \\
\hline Dong & 2012 & $103 / 97$ & 31 & 72 & $\mathrm{NR}$ & $\mathrm{NR}$ & 37 & 60 & $48 / 49$ & 13 & 35 & $\mathrm{NR}$ & $\mathrm{NR}$ & 29 & 20 \\
\hline He & 2012 & 48 & 13 & 35 & 27 & 21 & 27 & 21 & 105 & 31 & 74 & 72 & 33 & 73 & 32 \\
\hline Lilly & 2012 & 46 & 6 & 40 & $\mathrm{NR}$ & $\mathrm{NR}$ & $\mathrm{NR}$ & $\mathrm{NR}$ & 64 & 38 & 26 & $\mathrm{NR}$ & $\mathrm{NR}$ & $\mathrm{NR}$ & $\mathrm{NR}$ \\
\hline Li & 2012 & 31 & 8 & 23 & 8 & 23 & $\mathrm{NR}$ & $\mathrm{NR}$ & 19 & 8 & 11 & 5 & 14 & $\mathrm{NR}$ & $\mathrm{NR}$ \\
\hline Zhang & 2013 & $44 / 53$ & $\mathrm{NR}$ & $\mathrm{NR}$ & 24 & 20 & 17 & 36 & $18 / 21$ & $\mathrm{NR}$ & $\mathrm{NR}$ & 7 & 11 & 6 & 15 \\
\hline Li & 2013 & 29 & $\mathrm{NR}$ & $\mathrm{NR}$ & $\mathrm{NR}$ & $\mathrm{NR}$ & 9 & 20 & 73 & $\mathrm{NR}$ & $\mathrm{NR}$ & $\mathrm{NR}$ & $\mathrm{NR}$ & 46 & 27 \\
\hline Ji & 2014 & $19 / 24$ & $\mathrm{NR}$ & $\mathrm{NR}$ & 16 & 3 & 18 & 6 & $15 / 19$ & $\mathrm{NR}$ & $\mathrm{NR}$ & 9 & 6 & 16 & 3 \\
\hline Takako & 2014 & $17 / 16$ & 16 & 1 & 11 & 6 & 16 & 0 & $18 / 17$ & 18 & 0 & 8 & 10 & 15 & 2 \\
\hline
\end{tabular}

Abbreviations: FA, first author; T, tumor; N, lymph nodes; $\mathrm{S}_{1}$, stage I/II; $\mathrm{S}_{2}$, stage III/IV; NR, not reported.

Table 3: Positive/high Oct-4 and negative/low Oct-4 frequency in cases and controls: grade of differentiation

\begin{tabular}{|c|c|c|c|c|c|c|c|c|c|}
\hline FA & Year & & ases ( & itive/high) & & & ontrol & gative/low) & \\
\hline & & Total & Well & Moderate & Poor & Total & Well & Moderate & Poor \\
\hline Zhang & 2010 & 80 & 19 & 49 & 12 & 27 & 4 & 13 & 10 \\
\hline $\mathrm{Ge}$ & 2010 & 14 & 5 & 8 & 1 & 71 & 29 & 31 & 11 \\
\hline Chen & 2012 & 57 & 3 & 14 & 40 & 56 & 24 & 20 & 12 \\
\hline Dong & 2012 & NR & NR & NR & NR & NR & NR & NR & NR \\
\hline $\mathrm{He}$ & 2012 & 48 & 5 & 12 & 31 & 105 & 48 & 48 & 9 \\
\hline Lilly & 2012 & NR & NR & NR & NR & NR & NR & NR & NR \\
\hline $\mathrm{Li}$ & 2012 & 31 & 6 & 21 & 4 & 19 & 8 & 10 & 1 \\
\hline Zhang & 2013 & 53 & 13 & 19 & 21 & 21 & 13 & 5 & 3 \\
\hline $\mathrm{Li}$ & 2013 & 29 & 4 & NR & NR & 73 & 34 & NR & NR \\
\hline $\mathrm{Ji}$ & 2014 & 24 & NR & NR & 11 & 19 & NR & NR & 9 \\
\hline Takako & 2014 & 17 & NR & NR & 7 & 18 & NR & NR & 3 \\
\hline
\end{tabular}

Abbreviations: FA, first author; NR, not reported.

evidence of a significant association between positive/ high Oct-4 and cancer stage III/IV in different cancers. In addition, analyses stratified by primary tumor $\left(\mathrm{T}_{3-4}\right)$ and cancer grade of differentiation (intermediate-poor) showed a significant relationship with Oct-4.

The relationship of Oct-4 with cancer staging parameters suggests that positive/high Oct-4 can further inform the judgment of cancer malignancy based on basic TNM staging. Further large-scale investigations of Oct-4 and cancer stage are needed to confirm our results, but as additional evidence accumulates, Oct- 4 could prove to be an important biomarker for cancer malignancy. More importantly, in a total of 11 articles on Oct- 4 and cancer staging, there was only one paper related to analyzing the data in terms of Caucasian and the rest of the papers were Asian-related articles. The bewilderment that whether there were any possible bias related to racial issue seemed to need more time to consider. 


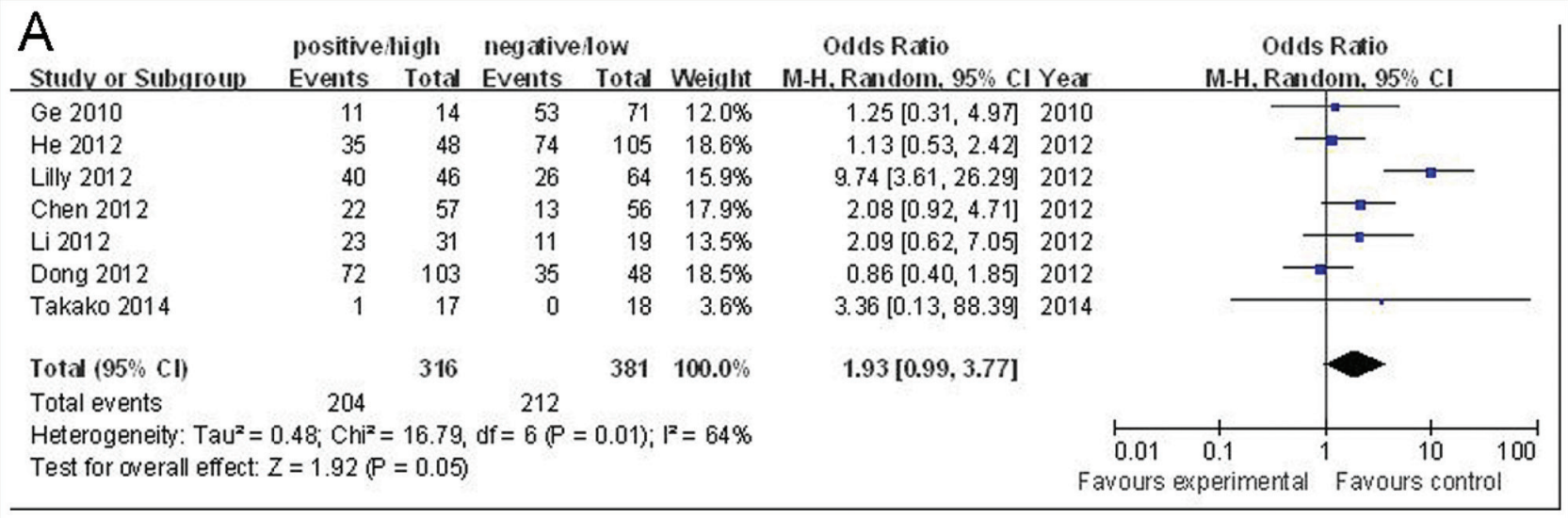

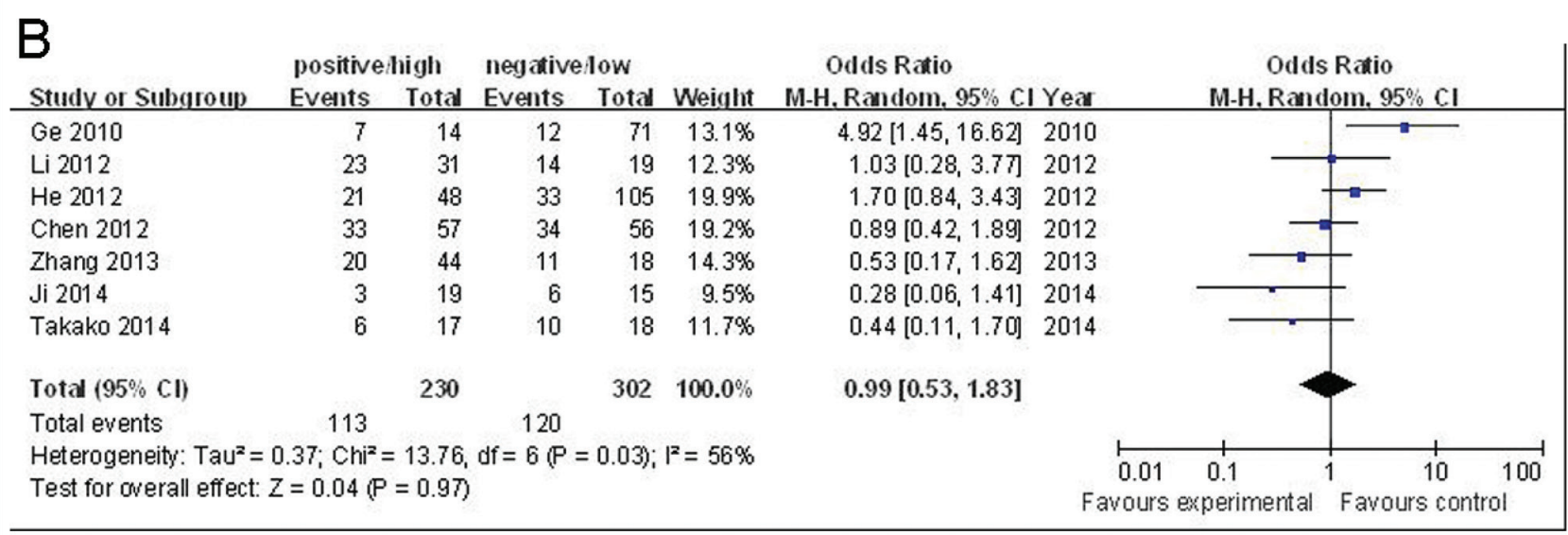

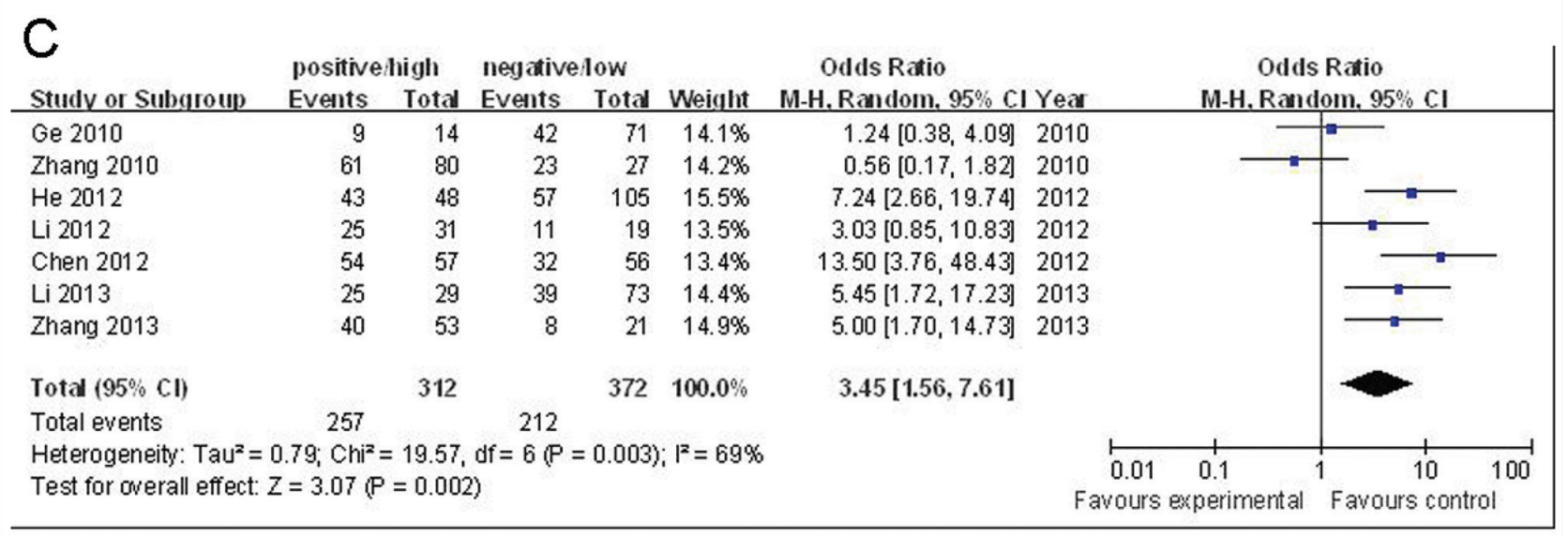

Figure 3: A. Forest plots of Oct-4 and primary tumor (T3-4) in all cases (positive/high Oct-4 vs. negative/low Oct-4). B. Forest plots of Oct-4 and lymph nodes (N1-2) in all cases (positive/high Oct-4 vs. negative/low Oct-4). C. Forest plots of Oct-4 and cancer grade of differentiation (intermediate-poor) in all cases (positive/high Oct-4 vs. negative/low Oct-4). The squares and horizontal lines correspond to the study-specific OR and 95\% CI. The area of the squares reflects the weight (inverse of the variance). The diamond represents the summary OR and $95 \%$ CI.

More importantly, several Oct-4 pseudogenes were recently reported to be transcribed in cancer cells. [39-43] For example, POU5F1B, a processed pseudogene that was highly homologous to Oct-4, was conferred an aggressive phenotype on gastric cancer and associated with poor prognosis. [39] OCT4-pg4, another pseudogene of OCT4, was found abnormally activation in hepatocellular carcinoma. [41] To date, by using RT-PCR and sequencing analysis, three Oct-4 pseudogenes, including OCT4-pg1, OCT4-pg3, and OCT4-pg4, were found to be expressed in some solid tumors, glioma, and breast carcer, from which cancer stem cells had earlier been isolated. [39-43] Interestingly, controversy existed that pluripotencyassociated isoform Oct4A was not expressed by malignant 
human urothelium, [40] and it was shown that some Oct-4 pseudogenes seemed be short of Oct-4 activities. Thus, whether Oct-4 expression in several cancer cells could actually be attributed to Oct-4 pseudogene expression remained unclear.

Some limitations of this meta-analysis should be noted. First, it was based on pooled positive or high Oct4 data; thus, we cannot separately assess the relationship of Oct-4 positivity and high-level expression of Oct-4 with cancer staging parameters. Second, the small-study effect, in which effects reported in small studies are larger, could not be avoided because in some cases the study population was relatively small $(<500)$. The small number of cases included in order to assess the relation of Oct-4 to other clinicopathologic parameters may set a limit to the conclusions drawn. As noted above, further large-scale studies with more detailed individual data are warranted to further validate the relationship between Oct-4 and cancer stage. Experiments as to the biological role of Oct- 4 in cancer progression and possibly a statistical analysis of a larger data set including an analysis of the types of cancers associated with Oct-4 expression would be helpful.

\section{MATERIALS AND METHODS}

\section{Publication search}

Computer searches were carried out using the following databases: PubMed, ISI Web of Knowledge, EMBASE, Google Scholar, China National Knowledge Infrastructure (CNKI), Chinese BioMedical Literature Database (CBM), and Wanfang database in China . The keywords were Oct-4, malignancy, neoplasm, cancer staging, and TNM staging. Articles published as of May 2015 that included case-control or cohort studies related to the association of positive/high Oct-4 with cancer stage were collected.

\section{Inclusion criteria}

The following criteria were used to select publications for further meta-analysis: (1) published in English and Chinese regardless of publication time; (2) evaluated associations between positive/high Oct-4 and cancer stage; (3) confirmed cancer patients pathologically; (4) included detailed cancer/TNM staging data; and (5) compared at least two groups (i.e., positive Oct-4 vs. negative Oct-4 or high Oct-4 vs. low Oct-4).

\section{Data collection}

Publication characteristic details, such as first author's name, publication year, patients' country of origin and ethnicity, total number of patients, cancer type, middle/ mean age of the study population and disease stage, were collected for each eligible publication. Positive or high Oct-4 and cancer/TNM stage was end points of interest.

\section{Statistical analysis}

Patients were divided into positive/high Oct-4 and negative/low Oct-4 groups. The associations between positive/high Oct-4 and cancer stage were determined by measuring odds ratio (OR) and associated 95\% confidence intervals (CIs). The significance of the pooled OR was determined by the $\mathrm{Z}$ test. Statistical heterogeneity among studies was assessed with Cochran's heterogeneity statistic, Q, and $I^{2}$, the latter of which describes variation that is due to heterogeneity rather than random error, as follows: $I^{2}=0-25 \%$, no heterogeneity; $I^{2}=25-50 \%$, moderate heterogeneity; $I^{2}=50-75 \%$, large heterogeneity; $I^{2}=75-100 \%$, extreme heterogeneity. [33] A fixed-effects model was applied in the initial analysis, and if significant heterogeneity existed, a confirmed random-effects model was used. Publication bias was evaluated using a funnel plot. All statistical analyses were carried out using Review Manager version 5.0 (Revman; The Cochrane Collaboration, Oxford, UK). All $P$-values in the meta-analysis were two-sided, and a $P$-value less than 0.05 was considered significant.

\section{CONCLUSION}

In summary, our meta-analysis provides evidence of an association between positive/high Oct-4 and cancer stage, especially in Asian. Thus, positive/high Oct-4 may play a role in contributing to higher-grade cancer. Further studies based on various cancer types are warranted to verify our findings.

\section{ACKNOWLEDGMENTS AND FUNDING}

This study was supported by grants from the Scientific and Technological Projects of Zhuhai City (No. 201310).

\section{COMPETING INTERESTS}

The authors declare that they have no competing interests.

\section{Author's contributions}

BZ, YL, and ZC conceived and designed the study; BZ, YL, YRL, and ZC participated in selecting the study, extracting data, performing statistical analyses and drafting the manuscript; FZ, XBZ, RJH, and WY were involved in critically revising the manuscript for important intellectual content. All authors read and approved the final manuscript. 


\section{REFERENCES}

1. Finicelli $M$, Benedetti $G$, Squillaro $T$, Pistilli $B$, Marcellusi A, Mariani P, Santinelli A, Latini L, Galderisi U, Giordano A. Expression of stemness genes in primary breast cancer tissues: the role of SOX2 as a prognostic marker for detection of early recurrence. Oncotarget. 2014; 5:9678-9688.

2. Zhong B, Wang T, Zou J, Zheng F, Huang R, Zheng X, Yang W, Chen Z. Association of the intermediate filament nestin with cancer stage: a meta-analysis based on 223 positive/high nestin cases and 460 negative/low case-free controls. Oncotarget. 2015; 26015397.

3. Lin Y, Zhong Y, Guan H, Zhang X, Sun Q. CD44+/CD24phenotype contributes to malignant relapse following surgical resection and chemotherapy in patients with invasive ductal carcinoma. J Exp Clin Cancer Res. 2012; 31:59.

4. Cirillo A, Di Salle A, Petillo O, Melone MA, Grimaldi G, Bellotti A, Torelli G, De' Santi MS, Cantatore G, Marinelli A, Galderisi U, Peluso G. High grade glioblastoma is associated with aberrant expression of ZFP57, a protein involved in gene imprinting, and of CPT1A and CPT1C that regulate fatty acid metabolism. Cancer Biol Ther. 2014; 15:735-741.

5. Burdon T, Smith A, Savatier P. Signalling, cell cycle and pluripotency in embryonic stem cells. Trends Cell Biol. 2002; 12:432-438.

6. Niwa H, Miyazaki J, Smith AG. Quantitative expression of Oct-3/4 defines differentiation, dedifferentiation or selfrenewal of ES cells. Nat Genet. 2000; 24:372-376.

7. Cheng L, Sung MT, Cossu-Rocca P, Jones TD, MacLennan GL, Jong JD, Lopez-Beltran A, Montironi R, Looijenga LH. OCT4: Biological functions and clinical applications as a marker of germ cell neoplasia. J Pathol. 2007; 211:1-9.

8. Kim JB, Greber B, Arauzo-Bravo MJ, Meyer J, Park KI, Zaehres H, Schöler HR. Direct reprogramming of human neural stem cells by OCT4. Nature. 2009; 461:649-653.

9. Ezeh UI, Turek PJ, Reijo RA, Clark AT. Human embryonic stem cell genes OCT4, NANOG, STELLAR, and GDF3 are expressed in both seminoma and breast carcinoma. Cancer. 2005; 104:2255-2265.

10. Atlasi Y, Mowla SJ, Ziaee SA, Bahrami AR. OCT-4, an embryonic stem cell marker, is highly expressed in bladder cancer. Int J Cancer. 2007; 120:1598-1602.

11. Liu CG, Lu Y, Wang BB, Zhang YJ, Zhang RS, Chen $\mathrm{B}, \mathrm{Xu} \mathrm{H}$, Jin F, Lu P. Clinical implications of stem cell gene Oct-4 expression in breast cancer. Ann. Surg. 2011; 253:1165-1171.

12. Ge N, Lin HX, Xiao XS, Guo L, Xu HM, Wang X, Jin T, Cai XY, Liang Y, Hu WH, Kang T. Prognostic significance of Oct4 and Sox 2 expression in hypopharyngeal squamous cell carcinoma. J Transl Med. 2010; 8:94.
13. Chen YC, Hsu HS, Chen YW, Tsai TH, How CK, Wang CY, Hung SC, Chang YL, Tsai ML, Lee YY, Ku HH, Chiou SH. Oct-4 expression maintained cancer stem-like properties in lung cancer-derived CD133-positive cells. PLoS One. 2008; 3:e2637.

14. Zhang X, Komaki R, Wang L, Fang B, Chang JY. Treatment of radioresistant stem-like esophageal cancer cells by an apoptotic gene-armed, telomerase-specific oncolytic adenovirus. Clin Cancer Res. 2008; 14:2813-2823.

15. Huang P, Qiu J, Li B, Hong J, Lu C, Wang L, Wang J, $\mathrm{Hu}$ Y, Jia W, Yuan Y. Role of Sox2 and Oct4 in predicting survival of hepatocelluar carcinoma patients after hepatectomy. Clin Biochem. 2011; 44:582-589.

16. Patrawala L, Calhoun $\mathrm{T}$, Schneider-Broussard R, Li H, Bhatia B, Tang S, Reilly JG, Chandra D, Zhou J, Claypool K, Coghlan L, Tang DG. Highly purified CD44+ prostate cancer cells from xenograft human tumors are enriched in tumorigenic and metastatic progenitor cells. Oncogene. 2006; 25:1696-1708.

17. Matoba R, Niwa H, Masui S, Ohtsuka S, Carter MG, Sharov AA, Ko MS. Dissecting Oct3/4-regulated gene networks in embryonic stem cells by expression profiling. PLoS One. 2006; 1:e26.

18. Park IH, Zhao R, West JA, Yabuuchi A, Huo H, Ince TA, Lerou PH, Lensch MW, Daley GQ. Reprogramming of human somatic cells to pluripotency with defined factors. Nature. 2008; Jan 10;451:141-146.

19. Hu TS, Liu SR, Breiter DR, Wang F, Tang Y, Sun S. Octamer 4 small interfering RNA results in cancer stem cell-like cell apoptosis. Cancer Res. 2008; 68:6533-6540.

20. Hu J, Qin K, Zhang Y, Gong J, Li N, Lv D, Xiang R, Tan X. Downregulation of transcription factor Oct4 induces an epithelial to mesenchymal transition via enhancement of $\mathrm{Ca} 2+$ influx in breast cancer cells. Biochem Biophys Res Commun. 2011; 411:786-791.

21. Zhang X, Han B, Huang J, Zheng B, Geng Q, Aziz F, Dong Q. Prognostic significance of OCT4 expression in adenocarcinoma of the lung. Jpn J Clin Oncol. 2010; 40:961-966.

22. Nan Ge, Huan-Xin Lin, Xiang-Sheng Xiao, Ling Guo, HuiMin Xu, Xin Wang, Ting Jin, Xiu-Yu Cai, Yi Liang, Wei$\mathrm{Han} \mathrm{Hu}$, Tiebang Kang. Prognostic significance of Oct4 and Sox2 expression in hypopharyngeal squamous cell carcinoma. Journal of Translational Medicine. 2010; 8:94.

23. Zhongyi Dong, Qin Zeng, Hesan Luo, Jinjin Zou, Chuanhui Cao, Jiyun Liang, Dehua Wu, Li Liu. Increased expression of OCT4 is associated with low differentiation and tumor recurrence in human hepatocellular carcinoma. Pathology Research and Practice. 208:527-533.

24. Chen Z, Wang T, Cai L, Su C, Zhong B, Lei Y, Xiang AP. Clinicopathological significance of non-small cell lung cancer with high prevalence of Oct-4 tumor cells. J Exp Clin Cancer Res. 2012; 31:10. 
25. He W, Li K, Wang F, Qin Y, Fan Q. Expression of OCT4 in human esophageal squamous cell carcinoma is significantly associated with poorer prognosis. World J Gastroenterol. 2012; 18:712-719.

26. Zhang Z, Zhu Y, Lai Y, Wu X, Feng Z, Yu Y, Bast RC Jr, Wan X, Xi X, Feng Y. Follicle-stimulating hormone inhibits apoptosis in ovarian cancer cells by regulating the OCT4 stem cell signaling pathway. Int J Oncol. 2013; 43:1194-1204.

27. Li XL, Jia LL, Shi MM, Li X, Li ZH, Li HF, Wang EH, Jia XS. Downregulation of KPNA2 in non-small-cell lung cancer is associated with Oct4 expression. J Transl Med. 2013; 11:232.

28. Ito $\mathrm{T}$, Sato $\mathrm{N}$, Yamaguchi $\mathrm{Y}$, Tazawa C, Moriya $\mathrm{T}$, Hirakawa H, Hayashi S. Differences in stemness properties associated with the heterogeneity of luminal-type breast cancer. Clin Breast Cancer. 2015; 15:e93-103.

29. Ji J, Wei X, Wang Y. Embryonic stem cell markers Sox-2 and OCT4 expression and their correlation with WNT signal pathway in cervical squamous cell carcinoma. Int J Clin Exp Pathol. 2014; 7:2470-2476.

30. Sobin LH. TNM: evolution and relation to other prognostic factors. Semin Surg Oncol. 2003; 21:3-7.

31. TNM Classification of Malignant Tumours . (5th ed.)WileyNew York: 1997; .

32. Zhao Y, Han Y, Zhang L, Wang Y, Ma Y, Zhang F, Fu D, Wang X. Quantitative assessment of the effect of cytochrome P450 2C9 gene polymorphism and colorectal cancer. PLoS One. 2013; 8:e60607.

33. Higgins JP, Thompson SG, Deeks JJ, Altman DG. Measuring inconsistency in meta-analyses. BMJ. 2003; 327:557-560.

34. Society AC. AJCC Cancer Staging Manual-Fifth Edition. Lippincott-Raven1997;
35. Wallin E, von Heijne G. Genome-wide analysis of integral membrane proteins from eubacterial, archaean, and eukaryotic organisms. Protein Sci. 1998; 7:1029-1038.

36. Gil J, Stembalska A, Pesz KA, Sasiadek MM. Cancer stem cells: the theory and perspectives in cancer therapy. J Appl Genet. 2008; 49:193-199.

37. Hochediinger K, Yamada Y, Beard C, Jaenisch R. Ectopic expression of Oct-4 blocks progenitor-cell differentiation and causes dysplasia in epithelial tissues. Cell. 2005; 121:465-477.

38. Chen SF, Lin YS, Jao SW, Chang YC, Liu CL, Lin YJ, Nieh S. Pulmonary adenocarcinoma in malignant pleural effusion enriches cancer stem cell properties during metastatic cascade. PLoS One. 2013; 8:e54659.

39. Hayashi H, Arao T, Togashi Y, Kato H, Fujita Y, De Velasco MA, Kimura H, Matsumoto K, Tanaka K, Okamoto I, Ito A, Yamada Y, Nakagawa K, Nishio K. The OCT4 pseudogene POU5F1B is amplified and promotes an aggressive phenotype in gastric cancer. Oncogene. 2015; 34:199-208.

40. Wezel F, Pearson J, Kirkwood LA, Southgate J. Differential expression of Oct4 variants and pseudogenes in normal urothelium and urothelial cancer. Am J Pathol. 2013; Oct 183:1128-1136.

41. Wang L, Guo ZY, Zhang R, Xin B, Chen R, Zhao J, Wang T, Wen WH, Jia LT, Yao LB, Yang AG. Pseudogene OCT4-pg4 functions as a natural micro RNA sponge to regulate OCT4 expression by competing for miR-145 in hepatocellular carcinoma. Carcinogenesis. 2013; 34:1773-1781.

42. Zhao S, Yuan Q, Hao H, Guo Y, Liu S, Zhang Y, Wang J, Liu H, Wang F, Liu K, Ling EA, Hao A. Expression of OCT4 pseudogenes in human tumours: lessons from glioma and breast carcinoma. J Pathol. 2011; 223:672-682.

43. Cantz T, Key G, Bleidissel M, Gentile L, Han DW, Brenne A, Schöler HR. Absence of OCT4 expression in somatic tumor cell lines. Stem Cells. 2008; 26:692-67. 